\section{How to nurse cats with lower urinary tract disease}

\section{Suzanne Rudd}

FLUTD can affect both male and female cats. Cats presenting with FLUTD can be obstructed or non-obstructed. Male cats most commonly present obstructed with a physical obstruction or urethral spasm, whereas females most commonly present with non-obstructive cystitis, usually feline idiopathic cystitis (FIC). FIC is the most common cause of FLUTD affecting both male and female cats. Physiologically there are some common abnormalities seen in cats with FIC. These include:

- Inappropriate hormonal and sympathetic nervous system response to stress

- Alterations in bladder neurons - neurogenic inflammation

- Reduced glycosaminoglycan (GAG) layer lining the bladder

- Compounds within the urine, increasing inflammation

FIC can be obstructive or non-obstructive. Obstructed cats must be seen and treated as an emergency to relieve the obstruction. Cats with non-obstructive FIC should be treated symptomatically, mainly by providing analgesia before investigating ways to prevent recurrence. Anti-spasmodics and anti-anxiolytic medications are also useful in cats with this disease.

Risk factors for FIC include:

- Chronic stress

- Acute stress

- Low water intake and concentrated urine

- Obesity

- Inactivity

- Indoor housing

- Use of an indoor litter tray.

Hospitalized cats should receive minimal handling in a low-stress environment. It is essential for the cat to be in a quiet area, have somewhere to hide and have easy access to food, water and the litter tray. Bladder palpation should be kept to a minimum and tableting medications should be avoided if at all possible.

The veterinary nurse can play an important role alongside the veterinary surgeon to help educate owners about FIC and discuss ways to provide a multimodal approach, which will decrease the chances of recurrence. In many cases behavioural advice may need to be considered, especially in cats that belong to a multicat household

\section{KEY LEARNING OBJECTIVES}

- To understand the causes of feline lower urinary tract disease (FLUTD)

- To understand the difference in treatment for urethral blockage versus urethral spasm

- To know how to talk to owners about feline stress

\section{MULTIPLE CHOICE QUESTIONS}

1. What is the most common cause of FIC?
(A) Low-fat diet
(B) Increased exercise
(C) Increased stress
(D) Diabetes mellitus

2. Which of the following statements is TRUE?
(A) Acute non-obstructive FIC usually lasts $<1$ day with a low rate of recurrence
(B) Acute non-obstructive FIC usually lasts 3-7 days with a low rate of recurrence
(C) Acute non-obstructive FIC usually lasts 3-7 days with a high rate of recurrence
(D) Acute non-obstructive FIC usually lasts 14-21 days with a high rate of recurrence

3. How can the recurrence of FIC be reduced?
(A) Decreasing stress
(B) Increasing water intake
(C) Reducing obesity
(D) All of the above

\section{Hepatic lipidosis}

\section{Sophie McMurrough}

$\mathrm{HL}$ is also known as fatty liver disease and is the most common acquired liver disease in cats. The condition is multifactoral and can be primary or secondary.

When anorexia is present, the body is starved of essential nutrients. The body automatically starts to break down other fat reserves to convert and utilize as energy. Cats are unable to break down large volumes of fat effectively, which leads to the liver becoming overwhelmed. Cats have a reduced ability to metabolize drugs and toxins which makes them more susceptible to liver damage. Even mild elevations in a cat's alkaline phosphatase (ALP) signifies a problem. ALP does not increase with steroids or hyperadrenocorticism in cats as it does in dogs.

\section{PRIMARY HL}

Primary $\mathrm{HL}$ is often seen in overweight cats. It is an acute hepatopathy caused by a large accumulation of fat in the hepatocytes. This leads to an acute loss of functioning hepatocytes which is reversible if the fats can be metabolized 